\title{
THE SENESCHAL ROSTAND DE SOLER'S REPORT TO EDWARD I ON THE NORMAN DEPREDATIONS IN SAINTONGE IN 1293
}

There are two almost identical versions of this report in the National Archives, London: $\mathrm{C} \mathrm{47/31/5/1} \mathrm{and} \mathrm{C} \mathrm{47/27/15/1.} \mathrm{Both} \mathrm{are} \mathrm{somewhat} \mathrm{damaged,} \mathrm{but} \mathrm{the} \mathrm{best}$ preserved is $\mathrm{C} 47 / 31 / 5 / 1$. Accordingly, I have used that version for the transcription. However, some parts are missing or are illegible, and I have used the $\mathrm{C}$ $47 / 27 / 15 / 1$ to complete the transcription. Only once have I digressed from the $\mathrm{C}$ $47 / 31 / 5 / 1$. This has been marked in a note. Variations are in the footnotes, and illegible words or passages have been marked with [...].

I have retained the original punctuation as far as possible. The starting letters of personal names and place names have been put in capitals, however.

"Excellentissimo principi et domino vero reverendo domino Edwardo dei gratia Regi Anglie domino Hibernie et Duci Aquitanie salutem Rostandus de Soler seneschallus Xantonie se ipsum ad pedes regis maiestatis ut prosperat sibi vota sucessus [...] ad [...] de mandato vestro per[...] michi facto de excessibus iniuriis et violentiis factis in terra [...] Xantoniensi vobis et gentibus vestris Angliis et aliis personis per Normannos ab inicio defensionis novissime[...] inter [...] et $[\ldots]$ pro $[. .$.$] quorum facta excessus a violentia perpetrata inferans anotan-$ tur quorum transcriptum domino [...] vel magistro Petro Aymerici clericis vestris misi Parisius secundum mandatum vestrum [...] predictas per presentiam petitionem.

Primo venerunt in prioratu Monasticii Novi $^{1}$ prope Sanctum Anianum ${ }^{2}$ qui est de obedientia justicia et superioritate vostra in terra Xantoniensis, Gaufridus Gossa magister navis vocate Larosa de Leura ${ }^{3}$ Godefredus Cormean magister cuiusdam navis de Berflers et Nicolas de la Mere magister cuiusdam navis de Berflers ${ }^{4}$ quarum nautum nomina non potui invenire cum decem et novem aliis Normanniis armatis videlicet actonis ${ }^{5}$ bacenetis ensibus balistis et aliis armaturis et in dicto prioratu intraverunt et de priore dicti loci per minas et verba iniuriosa habuerunt violenter contra voluntatem ipsius prioris unum sextarium bladi et duos pethesos ${ }^{6}$ que secum portaverunt

\footnotetext{
1 Montier Neuf.

2 Saint Agnant.

3 La Rose de Leure.

4 Barfleur.

5 Haketon. A light armour.

6 "Petasos"?
} 
Item ipsi malefactores vi rapuerunt et amoverunt quidam monacho Sola... Sancti Georgii de Esseron ${ }^{7}$ in dicto prioratu suam coram qua...gebatur et bursam suam in qua erat trigenti solidi turonensis et amplius que etiam secum portaverunt

Item predicti malefactores posuerunt quendam magnum cuttellum ad gurges cuiusdam veteris monachi dicti prioratus ac si vellent ipsum interficere qui audebat contradicere rapinis et violentiis quas faciebant in dicto prioratu

Item rapuerunt et secum portaverunt de dicto prioratu unam ballistam et baudrerium quos in dicto prioratu invenerunt et voluerunt interficere quondam servitorem dicti prioris nisi fugisset quia ausus fuit petere dictam balistam

Item cum quidam monachus dicti prioratus veniret ad ipsum prioratum obviavit ipsis malefactoribus quem ipsi malefactores suis vestibus exuere voluerunt et perquisierunt bursam suam et etiam in braccis suis invenerunt sex oboles(?) tantum in bursa dicti monachi quas rapuerunt et secum portaverunt

Item predicti malefactores intraverunt alia die in dicto prioratu manasteri Novi et invenientes dictum priorem ceperunt ipsum inter se et praeceperunt eidem quod redderet eisdem Robinum Anglicum Sancti Aniani et quosdam alios anglicos quos ipse receperat et reposuerat in dicto prioratu qui respondit quod dictus Robinus propter metum ipsorum Normannorum forte fugerat ad dictum locum et ibi per aliquos dies fuerat tamen recesserat de dicto prioratu et tunc iuverunt ad domum dicti Robino et quesiverunt domum suam utrum ipsum possent invenire quo non invento rapuerunt de domo predicti Robini barellis cal...[...] et alias mercaturas dicti Robini que omnia secum portaverunt et camisiam ancille dicti Robino rapuerunt et portaverunt. Et hec facta fuerunt circa Octavas festi Pasche proximi preteriti anno domini M CC nonegesimo tertio.

Item predicti Nicolas de la Mere cum multis aliis Normanniis complicibus suis intraverunt per [...] in domo Gyletini Ulbaudi in terra vestra Xanctoniensi et quandam arcam ipsius Guillermi(!) fregerunt de qua extraxerunt unum monile et quendam annullum argentos panes caseos et alia que omnia secum portaverunt.

Item ille Nicolas de la Mere et suis complicibus armati intraverunt in prioratu de Podio [...] in terra vostra existentes et ceperunt per violentiam de [...] dicti prioratis bona $[. .$.$] quem secum portauerunt [...] fuerint cum dicto priore contra$ voluntatem suam.

Item predictus Nicolas de la Mere fuit et suis complicibus Normanniis armati intraverunt domino Guillermi(?) Begandi(?) huius [...] et eis rapuerunt duodecim $[. .$.$] quos habebat in bursa quid plures [...] ipsos duodecim denarii parisis.$

Item et $[. .$.$] fuerit intra predictum festum Pasche et sequentem [...]$

Item venerunt plures Normanni usque ad viginti et amplius armati loricis batonis et sagittis ensibus et aliis [...] ad domum Rectoris ecclesie Sancti Nazarii ${ }^{8}$ prope Subisiam ${ }^{9}$ que est de obediencia et superioritate vestra die domenica quindem Pasche proximo preterito et cum intrassent domum dicti presbiteri

\footnotetext{
7 Unknown location. Possibly Saint-Georges-des-Coteaux.

8 Saint-Nazaire-sur-Charante.

9 Soubise.
} 
ipse presbiter eos ad prandium invitavit et dedit eis panem vinum caseum et alia cibaria que tunc habebat parata et cum pransi fuerunt dictus presbiter credens quod ipsi recederent exuere domum suam et cum exisset malefactores ceperunt capones et galinis quos capere potuerunt et sic cum dictis galinis et capunibus recesserunt

Item ipsi [...] cum multis aliis armati iterum ad domum dicti presbiteri venerunt die martis sequente post quindenam Pasche [...] dicto presbitero rapuerunt et ceperunt de domo dicti presbiteri omnes galinas capones anseres et porcellos que dictus presbiter habat in domo sua nec unum caponem vel galinam dimiserunt et secum premissa portaverunt

Item die Jovis proxima post quindenam Paschi venerunt iterum alii malefactores Normanni non illi qui supra venerunt usque ad quartuor decim [...] et cum dictus presbiter sensisset ipsos venientes absentavit se timens de malicia eorundem et resecavit cum magnas barros prout melius potuit ostium camere sue et exivit per quandam fenestram et fugit in viridarium suum et intravenientes ipsi malefactores in domum dicti presbiteri petierunt ab ancilla sua ubi erat dictus presbiter que respondit quod nesciebat et statim venientes ad ostium camere dicti presbiteri fregerunt dictum ostium et ibidem intraverunt et fregerunt quendam forcerium de quo quemdam annulum aureum cum bono lapide precioso valore centum solidorum et quedam alia jocalia argenta rapuerunt ceperunt et portaverunt et etiam cartas memoralia et alias litteras in dicto forcerio existentes [...] ceperunt et portaverunt et cum ipsi hoc fecissent intrantes viridarium dictum presbiterum invenerunt et arcubus et ensibus et sagitiis super positis venerunt maliciose contra ipsum et ipsum tanquam latronem ceperunt et captum in sua camera adduxerunt que eum inter ipsos malis suis gradibus sedere fecerunt quo sedente preceperunt ei quod aperiret arcas suas et antequam posset respondere [...] stupefactus aliqui ex ipsis dictas arcas statim frangere voluerit cum securibus et aliis ferramentis que ibidem invenerunt et sic [...] oportuit miserimo presbitero arcas suas aperire quibus apertis ipsi raptores ceperunt sextaginta et decem solidos et amplius lintheamina robas et vestes ipsius presbiteri et vestimenta illa que pueri receperunt in baptismia et multa alia bona ipsius presbiteri que erant in dictis arcis [...] et portauerunt

Item rapuerunt et secum portaverunt predicti raptores de domo ipsius presbiteri multa ferramenta et bonos videlicet ferreos ligones ${ }^{10}$ et martellos et cutellos ferreos et quandam furcam ferream et alia quem[...] voluerunt et in recessu ${ }^{11}$ [...] dicto presbitero et perceperunt quod eisdem centum libras turonensis mutaret alioquin sciret se condempnari sententia capitali postmodum [...] vero primo die maii sequentis venerunt ad domum dicti presbiteri decem Normanni alii quam predicti $[. .$.$] presbiter eos videret intrantes ipsos plus per timore quam$ pro dilectione motus ad prandium invitavit et eisdem vini [...] clara et rubea et clarea que tunc habebat parata dedit eis quibus sic pransis ipsi ducentes dictum

10 “videlicet(?) ferreos(?) ligones(?)," from TNA C 47/27/15/1. This passage is illegible in TNA C $47 / 31 / 5 / 1$.

11 "in recessu," from TNA C 47/27/15/1. This passage is illegible in TNA C 47/31/5/1. 
presbiterum [...] requisierunt cum ${ }^{12}$ quod mutaret vel daret eis viginti librarum turonensis alioquin ipsum capite privarent et ignem ponerent [...] domo suo [...] responsavit quod ${ }^{13}$ denarios non habebat quia alii Normanni qui predicto die Jovis venerunt ceperant omnia bona [...] et secum recesserunt viros(?) extra villam $^{14}$ ad crucem ville et ibidem remanserunt et residentes ibidem dicto presbitero mandaverunt per quendam ${ }^{15}[. .$.$] ipsi Normanni { }^{16}$ reneverunt ad dictam villam Sancti Nazarii cum tabardis in brachii involutis et cum [...] domum dicti presbiteri [...] intraverunt et invenientes dictum presbiterium a quendam clericum extravenum qui ibidem venerit ${ }^{17}[\ldots]$ pro ipso presbitero visitando eidem clerico quendam ensem quem portabat subtus super secum amoverunt et postea $[\ldots]$ ipsam violentiam [...] ipsum vestribus suis quibus inductus erat exuere voluerunt quem cum ita exuerunt gentes que viderunt dictum factum clamaverunt erunt aus[...] entia cui clamore venerunt gentes de patria et ipsos malefactores fugaverunt et aliquos ex ipsis [...] vulnaverunt In Crastino venerunt ad dictam villam centum quinquaginta vel plures de dictis Normannis armatis Lanceis balistis ensibus et aliis diversis armatis et domum dicti presbiteri intraverunt et decem tonellos vini boni et puri fregerent et tirculos(?) tiderunt(?) et totum vinum ad terram fuderunt necnon plures alios tonellos grossos archas tabellas bacinetos et omnia utensilia et superlectulia domus dicti presbitieri et ruperunt fregerunt et penitus destrugerunt quod plura quod detestabile est sacrilegium commitentes portas ecclesia sue fregerunt et archas ipsius eclesiae aperientes frangentes monumentis et ornamentis ecclesiastitis ut pote(?) mappis altaris et aliis ornamentis ipsam ecclesiam privaverunt et quod deterius et oribile corpus Christi et sanctam eucharistiam ceperunt de dicta ecclesia et luminaria dicte ecclesie et cimbala de clocherio descenderunt et quandam ymaginem beate Mariae Virginis de ecclesia extraxerunt et secum omnia premissa in vituperium Dei et sancte ecclesie et in suarum omniarum dispendium portaverunt et preter hec predictus Gaufridus Gossa magister navis vocati Larosa sancta eucharistia usus fuit ipsam ut dicitur et fama referet corporaliter in sue detrimentum anime corporaliter masticando.

Item post modum quadam alia die venerunt alii malefactores de Normannis predictis ipso presbitero absente de suis partibus que fugerat ad partes alienas propter metum dictorum Normannorum ad domum dicte presbiteri et duos boves arantes et unum rucineum et instrumenta ferrea ad faciendum ostias ${ }^{18}$ et pluria alia feramenta rapuerunt et secum portaverunt et sic dampnificaverunt ipsum bene usque ad valorem centum quinquaginta librarum nec non in talem statum dictum presbiterum posuerunt quod non habet modo dictus presbiter sustentationem necnon provusionem suam et omnia ista facta fuerunt in terra vestra Xantoniensis.

12 "requisierunt cum," from TNA C 47/27/15/1. This passage is illegible in TNA C 47/31/5/1.

13 "responsavit quod," from TNA C 47/27/15/1. This passage is illegible in TNA C 47/31/5/1.

14 "et secum recesserunt viros(?) extra villam," from TNA C 47/27/15/1. This passage is illegible in TNA C $47 / 31 / 5 / 1$.

15 "per quendam," from TNA C 47/27/15/1. This passage is illegible in TNA C 47/31/5/1.

16 TNA C $47 / 27 / 15 / 1$ has "statim" instead of "Normanni".

17 from TNA C 47/27/15/1. This passage is illegible in TNA C 47/31/5/1.

18 TNA C 47/27/15/1 has "hostias". 
Item venerunt $[\ldots]$ malefactores Normanni ad molendinos Prioris de Subisia in vestra terra Xantonensi et ferra seu instrumenta ferrea dictorum molendinorum ceperunt et asportaverunt secum molas dictorum molendinorum ad terram prostraverunt.

Item fuerunt multi malefactores de ipsis Normannis de nocte in domo Johannis Gauteri morantis ${ }^{19}$ prope Subisiam in terra vestra et quendam Pethesum et quinquaginta libras parvi linei de ipsa domo contra voluntatem ipsius rapuerunt et secum portaverunt de hominibus ipsorum malefactorum certiorari potuerunt nullo modo.

Item fuerunt multi malefactores de ipsis Normannis in domo domini Arnaldi Boterelli presbiteri de nocte in terra vestra Xantonensi et de domo ipsius presbiteri gallinas capones ex multa alia bona dicti presbiteri rapuerunt et secum portaverunt et dictum presbiterum nudum fugientem et clamantem cum ensibus fugaverunt nomine vero illorom malefactorum penitus ignorantur.

Item ipsi malefactores fregerunt plures tonellos Petri Bernardi de Letelon' mansionis vestre et vinum in dictis tonellis contentum ad terram fuderunt et mayramentum(?) dictorum tonellorum et etiam archas suas tabellas et multa alia bona sua combusserunt ipsum dampnificando usque ad valorem triginta librarum et amplius.

Item rapuerunt et per violentiam amoverunt in una publica terra vestre Xantonensis cuidam homine nomine Guillermo Bardelli tres solidos de bursa sua.

Item rapuerunt cuidam alteri homine nomine Perotus Mercerius septem para caligarum et quedam alias merces suas et quadraginta solidos in pecunia et istud factam fuit per dictum mercatorum expositum Constabulo custodientibus navigium Normannorum qui fecerunt pecuniam tantam et residuum sibi retinuerunt.

Item venerunt de dictis Normannis in domo Johannis de Peilhe et invenientes quondam filiam suam petierunt ab ea ubi erat archa in qua erant denarii dicti patris sui qui dixit quod nesciebat et statim apprehenderunt eam et prostraverunt ad terram et quandam magnam tabulam super eam posuerunt et postea duo ex ipsis super ipsam tabulam sederunt ipsam filiam deprimentes et male tractantes et cum propter huiusmodi oppressionem non possent ab ea scire ubi erant denarii patris cur dimiserunt eam tamen de bonis dicti Johannis que in domo ipsius invenerunt ceperunt et secum portaverunt ad valentiam triginta librarum

Item venerunt multi malefactores de dictis Normannis in villa Sancti Anyani prope Subisiam que movet de feodo vestro et invenientes quandam mulierem tenentem puerum inter brachia sua petierunt ab ea cuius erat dictus puer asserentes ipsum sue filium anglici que dixit quod non erat Immo erat filius suus et cuiusdam viri sui nati de patria et statim unus ex ipsis respondit dicens tu mentiris iste puer non est tuus quia modo tenebas alium puerum inter ulnas tuas et tunc respondit ipsa mulier quod duos pueros habuerat et peperat (sic!) insimul istum et alium et tunc unus ex ipsis irruens inter ipsam mulierem voluit sibi rapere dictum puerum sed ipsa mulier de puero amplexato prostravit se ad terram cum dicto puero clamando quod dictum puerum interficerent volebant

19 "morantis," omitted in TNA C 47/27/15/1. 
Normanni et congregata multitudinem gentium clamorem dicte mulieris ipsi malefactores recesserunt.

Item venerunt apud Mignanes ${ }^{20}$ in domo cuiusdam boni viri et invenientes ipsum cum quadam sua filia ipsam filiam ceperunt et ipsam carnaliter per violentiam tres vel quatuor ex ipsis cognoserunt in presentia patris sui et quia ipse pater ausus clamare ipsum atrociter verberaverunt et male tractaverunt

Item venerunt ad domum Guillermi Caradelli valeti et invenientes uxorem ipsius valeti eam cognoscere per violentiam carnaliter voluerunt nisi aliqui coadiutores superuenissent qui dictam mulierem defenderunt et eam de manibus eorum liberaverunt quam mulierem postea statim extra patrum feci duci propter timorem ipsorum malefactorum.

Item venerunt in prioratu Sancte Gemma ${ }^{21}$ sex Normanni cum ensibus petentes et querentes Anglicos qui fugerant ibidem et requisierunt gentes dicti prioris quod Anglicos ibidem repositos traderent et libarerent eisdem alioquin quod dampna facerent in prioratu predicto.

Item ceperunt capelanum de Cerayo $^{22}$ mansionarium in terra vestra Xantonensi et captum eum duxerunt apud Sanctum Savinianum ${ }^{23}$ in manibus suis et captum tenuerunt quousque fuit per predictos Constabulos Navigii liberatus pro eo autem captus fuit quia se advocavit et mansionarium in terra vestra.

Item die sabati ante festum beati Gregorii proximo preteritum venerunt multi Normanni in Civitate vestra Xantonensi ${ }^{24}$ cum ensibus succinctes et $[. .$.$] ... tes$ per dictam civitatem petebant cuius erat illa civitas respondentes gentes dicte civitatis dicebant quod domini Regis Anglie et tunc succuciebant et movebant capita sua postmodum vero petebant qui erant ditiores homines de civitate. Et respondentes gentes nominabant illos qui magis in diuities habundebant et inquirentes domos et mansiones ipsorum faciebant signa in domibus eorumdem et aliquas signabant in ostiis et aliquas in fenestis et dixerunt multotiens publice per civitatem quod ipsam in vituperium domini Regis Anglie comburerent et destruerent accidit autem die Jovis sequente ante dictum festum Sancti Georgii quod plures Normanni venerunt ad dictam civitatem portantes secum aliqui enses ffauchones ${ }^{25}$ et petierunt domum cuiusdam boni viri mercatoris de civitate Anglici nomine magister Galterus Anglicus qui habet et habuit per longa tempora mansionem in ipsa civitate una cum uxore liberis et familia sua et cum pervenissent domum dicti mercatoris invenerunt ipsam domum clausam et bene reseratam quia ipse mercatore multum timebat de ipsis Normannis pro dampnis et injuriis que et quas aliis inferebant et sederunt ante portam dicte domus et respicientes non viderunt locum per quem intrare possent in dicta domo et tunc accedentes ad ipsam januam sub [...] eam fortiter ac si vellent ipsam frangere quod bene facere temptaverunt hoc videns autem quidam homo de civitate cum

\footnotetext{
20 Unknown location.

21 Sainte-Gemme.

22 Unknown location.

23 Saint-Savinien.

24 Saintes.

25 Falchion. A heavy one-edged sword.
} 
multis aliis astantibus petiit ab eisdem quid vellebant et respondentes dixerunt quod aportarent pecuniam dicto magistro Galtero quam volebant sibi solvere et tunc respondit eisdem dictus homo quod ipse non erat tunc in domo nec etiam in villa et statim dictam januam magis [...] volentes ibidem intrare si possent et tunc dictus homo dixit eis quod male faciebant quo dicto unus ex ipsis extraxit ensem et voluit ipsum percutere et cum dictus homo videret gladium contra se evaginatum clamavit fugiendo per villam aus murtrerios ${ }^{26}$ Normannos et tunc dicti omnes Normanni illi extraxerunt enses suas propter quod omnis civitas fuit commota et clamabant omnes aus murtrerios Normannos. Dicti vero Normanni aliquos de civitate cum ensibus et aliis gladiis percusserunt. Et cum ego predictus Rostandus de Solerio audirem tantum tumultum in civitate misi gentes meas ibidem videre quid hoc erat et venientes dicte gentes meas versus ipsos Normannos invenerunt ipsos cum ensibus fauchonibus et aliis gladiis evaginatis et precipientes eisdem Normannis quod redderent eis dictos enses et ceteros gladios dixererunt quod non facerent Immo fuerunt in defensa et voluerunt vulnerare gentes meas et tunc gentes mei evaginatis gladiis suis ceperunt octo de ipsis Normannis quorum duos vulneraverunt quia aliter capi non potuerunt et ipsos ad me in castro Xantonensi captos adduxerunt ceteri vero fugerunt et cum ego dictus Rostandus viderem duos ex ipsis vulneratos statim feci perquiri cirugicos et preparere plagas eorum et inquisita veritate qui ex ipsis erant in culpa dicti facti inveni ipsos duos vulneratos culpabiles quos in prisona retinui in Castro Xantonensi et sex alios eorum consocios quos cum parva culpa inveni liberavi et eis reddi feci enses suos et alia bona sua in crastinum vero facta congregatione et conspiratione inter ipsos et alios Normannos navigii qui erant apud Sanctum Savinianum inter se ordinaverunt quod cum quatuor milibus armatorum venirent aggredi civitatem et ipsam igni submittere et totaliter destruere et gentes interficere si possent que conspiratio per aliquos amicos dicte civitatis fuit michi et burgensibus dicte civitatis nunciata et tunc omnes de civitate perteriti et stupefacti fugerunt huc et illuc cum bonis suis cum non haberent arma cum quibus se defendere possent. Ego vero Rostandus accidi [...] et quod ... tebatur feci muniri et dictum castrum prout melius potui licet parum vel quasi nichil invenirem ad defensionem castri si dictus casus accidisset et porta Civitatis claudi et custodiri cum magni expensis ipsa vero die veneris semper ${ }^{27}$ expectante adventum et insultum dictorum Normannorum miserunt ad me Constabuli navigii predicti plures servitores domini Regis Francie Normannos et maiores magistros navigii post ipsos Constabulos et venerunt ad januam castrum Xantonensem requierentes januam quod permiterret eos intrare in castro quia mecum volebant loqui ex parte domini Regis Francie et dictorum Constabulorum quos precepi in castum recipere et cum intrassent $[\ldots]^{28}$ ex ipsis nomine C... [... $]^{29}$ domini Regis fuit $[\ldots]^{30}$ me ex parte domini Regis Francie constabularie custodientii navigii Normannorum quod duos [...]

\footnotetext{
26 "Aux meurtres!"

27 TNA C 47/27 "semper me".

28 Illegible in TNA C 47/31 and omitted in TNA C 47/27/15/1.

29 Illegible in TNA C 47/31 and omitted in TNA C 47/27/15/1.

30 Illegible in TNA C 47/31 and omitted in TNA C 47/27/15/1.
} 
quos captos tenebam in castro Xantonensi qui ad partes Xantonensi se venebant sub gardia et protectione domini Regis Ffrancie eis redderem et a dictis prisona ${ }^{31}$ liberarem cum cognitione ipsos si in aliquem commissem spectaret in mediate ad dominum Regem Ffrancie vel ad gardiatores dicti navigii ratione gardie antidicte Ego vero vocatu meum Consilium venerabili Decano et pluribus canonibus Xantonensibus multis hominibus ligeis vestris nobilibus et alii meliori predicte civium Xantonensi retuli et explicavi eisdem servientibus causam quare dicti Normanni capti fuerant et arrestati et modum captionis et totum processum superius nominatum et dixi quod vos non suberratis nec gentes vestros suberrant mandato alicuius iuditis vel delegati curie Ffrancie nisi ipsis expresse habebant certum mandatum a dicta curia et requisivi ab eisdem mandatum exhiberi si super hoc haberent quod non habebant plures ratione eficaces propositii ${ }^{32}$ quare dictos captos liberare non tenebar nec propter requestam predictam ipsos volui liberare. Et audientes quod dictos captos liberare nolebam statim perceperunt michi ${ }^{33}$ ex parte [...] domini Regis Ffrancie sub incurrimento bonorum meorum quod eisdem dictos captos liberarem cuius precepto nullatenus voluit obedire dixi tamen dicti presentibus ${ }^{34}$ ne forte viderent latrones fuisse pensari vel maliciose contra ipsos Normannos quod peius vel similie fecissem personis aliis si taliter volnerassent ${ }^{35}$ quod ob reverentiam dicti domini Regis Ffrancie paratus eram eos liberare hac Conditione adiecta et et protestatione expresse facta quod ipsos malefactores totaliter tenerent et imprisonarent quod de violentii et excessibus commissis in dicta civitate possent vobis et vestris gentibus et passis iniuriam satisfactione facere ${ }^{36}$ et etiam emendare quotiens essent super hoc requisiti et cum dicti capti fuerant liberati sub conditione predicta

Item rapuerunt et secum portaverunt quindam de Normannis predictis violenter de bonis Arnaldi Bartholomei mansionaris in terra vestra Xantonensi tam in bladis quam panineis et rebus aliis ad estimationem ad decem librarum turonensium

Item de bonis Bartholomei de Fonte Regnialde ad valorem sexta librarum et ulterius et voluerunt ipsum interficere et exuerunt ipsum vestibus suis

Item habuerunt rapuerunt et secum portaverunt de bonis Johannis Chant per violentiam ad estimatione sextaginta solidorum et amplius.

Item habuerunt rapuerunt et secum portaverunt domini Guyllermi de Paugues duo dolea vini et ultra fuderunt ad terram maiorem partem alterius tonelli et hoc fecerunt de nocte.

Item habuerunt per violentiam de bonis Prioris de Subisia tam in blado vino denariis ciphis argenteis pannis et rebus aliis ad valorem quatuor decim librarum.

31 "prisones," in TNA C 47/27/15/1.

32 TNA C 47/31/5/1 has "eorum [...] Ffrancie propter sui" but this makes less sense than "ratione eficaces propositii" in TNA C 47/27/15/1. Thus, I have preferred this reading.

33 TNA C 47/27/15/1 has "in" not "michi".

34 Almost illegible.

35 TNA C 47/27/15/1 has "deliquissent", not "volnerassent".

36 "facere" omitted in TNA C 47/27/15/1. 
Item habuerunt de bonis Petronille Vesuerie duo dolea vini per violentiam et contra voluntatem suam.

Item de bonis Petri Trolli ${ }^{37}$ in pannis utensilibus et aliis ad valorem vinginti solidorum.

Item de bonis Petri Calibere tam in pethesis blado vino et aliis rebus ad valorem triginta solidorum.

Item de bonis Johannis Bernardi tam in blado vino denariis et rebus aliis ad valeriam decem librarum et amplius ulterius quod fuderunt ad terram vinum dicti Johannis ad estimationem dimidii tonelli.

Item rapuerunt quidam presbitero nomine dominus Hugo Guyllermi octo decim solidos quos habebat in bursa sua et ceperunt eum in publica via ${ }^{38}$ et eidem multa viturperia fecerunt cum Constabularius navigii fecerunt sibi reddi duo decim solidos et sexta solidos duos retinuerunt.

Item rapuerunt et per violentiam portaverunt de domo Johannis Reginaldi quartuor decim lintheamina et quinque pulvinaria et duo coopertoria furrata pellibus toni coloris et duas mappas meniedeyngnias (sic!) et unam calderiam et unum vestimentum cum quo missa celebratur et fregerunt portam domus sue que omnia estima valere poterant centum solidos

Item rapuerunt et secum portaverunt de domo dictorum Aus Quasbenes (sic!) duodecim costeretos boni vini et decem costeretos boni de reffon(?) et tabulas dicte domus et archas suas fregerunt et exinde duodecim bussellos bladi extraxerunt et portaverunt

Item intraverunt in domo Johannis Liore et rapuerunt et secum portaverunt dimidium pethesum et duos agnos.

Item intraverunt et rapuerunt de domo Arnaldi Arguyn duos porcellos et alia bona dicti Johannis ad valorem quadraginta solidos

Item intraverunt et rapuerunt de domo Johannis Ogardi dimidium pethesum et fuderunt ad terram unam tonellum vini alia bona dicti Johannis ceperunt ad valorem decem librarum.

Item cum duo mercatores de Navarre bone persone et divites venirent de Rupella $^{39}$ apud Burdegalem ${ }^{40}$ progressuri pro suis mercibus ${ }^{41}$ et facerent transitum per villam Sancti Saviniani predicti Normanni cum maxima multitudine ipsos mercatores ceperunt credentes ipsos esse Bayonenses et ipsos ceperunt et octo milia Florinorum eisdem in primis rapuerunt et postmodum eos viliter occiderunt et frustarunt [...] eosdem nec mors nec membrorum decisio potuit eis sufficere Immo revera quidam ex ipsis Normannis posuit qui astavit in quadam lancea intestina dictorum mercatorum et portabat ad collum per villam sancti Saviniani dicens quis vult emere Trypes de Bayona ego vendam et quidam alius de ipsis Normannis cepit caput alterius defunctorum et auriculam ipsius cum dentibus coram toto populo masticabat et maximim partem ipsius auriculae de

37 TNA C 47/27/15/1 "Trolii".

38 TNA C 47/27/15/1 "via publica vestra".

39 La Rochelle.

40 Bordeaux.

41 TNA C 47/27/15/1 "mercibus procurand". 
capite emulsit et comedit quod inhumaniter fuit factum et pernitiosum exemplo maxime quia constabul'(?) et custodes navigii antedicti presentes in villa et hoc scientes vendicare comtempserunt quibus sic peractis membra ipsorum interfectorum postmodum in flumen Carantonis ${ }^{42}$ proieterunt

Item venerunt ad domum Rectoris ecclesie de Agonay ${ }^{43}$ et super muros clamose dicte domus ascendentes in ipsa domo intrare voluerunt et cum capellanus seu Rector predictus hoc videret venit contra eos petens ab eis quid volebant qui dixerunt quod statim sciret et descenderunt infra dictam clausuram et cum dictus presbiter videret ipsos descendentes fugit in domum suam et portam domus sue cum barris et cum aliis ingeniis prout melius potuit reseravit et insequentes ipsum portas ipsius domus fregerunt et presbiterem predictum quem invenerunt statim ceperunt precipientes eidem quod statim archas suas aperiret quod facere denegavit et frangentes archas de ipsis extraxerunt et rapuerunt undecim libras turonenses, et septem viginti turonenses grossos argenteos et unum tabardum de perso forratum et unum ensem et tres ciphos argenteos et duo decim clocheria argentea et multa alia bona ad estimationem quinquaginta librarum.

Item violenter rapuerunt ceperunt et secum portaverunt de domibus personarum infra scriptarum bona que secuntur videlicet de domo Johannis de Airolio quartuor decim denarios et voluerunt portare lectos et cooperturas lectorum suorum et mantellum uxoris sue.

Item de domo dicti Johannis qua quatuor caseos et duas gallinas

Item de domo Benedicti Taste Crispe unum capriolum et duas gallinas.

Item de domo Petri Suttoris duas gallinas.

Item de domo dictorum Elyars quartam partem unius pethesi

Item de Prioratu de Romegus ${ }^{44}$ quatuor tibias porcii

Item de domo Laurentis de Boyes unum annulum argenteum

Item de domo Aymerici Vignerii unum supertunica [...] quatuor Lintheamina

Item de Gaufrido Ossendi unam gallinam de Gaufredo Achardi unam gallinam de Vigerio de Melay unum capriolum de Arnaldi prepositi unum agnum et unum porcellum et quartuor caseos et quartam parte unius Pethesi

Item de Arnaldo Granerii duas gallinas et duos caseos et de domo dicte Alaseguine ceperunt vinum quantum ibi invenirunt ad estimationem decem corperellorum et illud consumpserunt

Promissa autem omnia et multa alia forefacta comissa fuerunt per dictos Normannos in terra vestra Xantonensi et locus circum vicinis inter festum Pasch' proxime preteritum et sequentem festum Ascensionis Domini prout per relationem et testimonium personarum predictarum inquisitarum et prout fama patria attestatur vero nomina illorum exceptis quibusdam superius nominatis penitus ignorantur nam quidam veniebant una die et alii alia die sic vicissim ne possent discerni ab aliquo vel cognosci.

Datum Xantonis quinto die mensis julii anno Domini mcc nonagesimo tertio."

\footnotetext{
42 The Charente River.

43 Agonnay.

44 Romegoux.
} 